UCTP104.96

\title{
The Structure of Space-Time Emerging from the Two-Superbody Problem in Chern Simons Supergravity
}

\author{
Sunme Kim \\ Physics Department, University of Cincinnati, Cincinnati, OH 45221
}

\begin{abstract}
We show that the exact solution of the two-superbody problem in $N=2$ Chern Simons supergravity in $2+1$ dimensions leads to a supermultiplet of space-times characterized by the two gauge invariant observables of the super Poincaré group. The metric of this space-time supermultiplet can be cast into the form of a spinning cone in which the coordinates do not commute or of a spinning cone with an additional finite discrete dimension. Some of the interesting features of this universe and their possible physical implications are discussed in the light of a corresponding observation by Witten.
\end{abstract}

*e-mail address: skim@physunc.phy.uc.edu

†e-mail address: mansouri@uc.edu 
It has been known for sometime that supergravity theories in $2+1$ dimensions can be formulated as Chern Simons gauge theories of the corresponding supergroups [1-4]. The main focus of the present work is to explore the physical properties of the emerging space-time when supersymmetric matter is coupled to these theories in a super Poincaré gauge invariant manner. Although much of what we describe is applicable to any supergravity theory, for definiteness we will consider the $N=2$ Chern Simons gauge theory of the super Poincaré group.

It has been pointed out recently that the two-superbody problem in $N=2$ Chern Simons supergravity is exactly solvable [5]. We will give below a physical interpretation of this solution and show that it possesses a supersymmetric space-time structure [6]. In arriving at this conclusion, one of the issues we will address is the question of the asymptotic observables associated with such a supersymmetric space-time. In this connection, Henneaux has analyzed the metrical field theory of supergravity (without matter) in $2+1$ dimensions [7]. Assuming that the space-time is asymptotically conical, he concludes that, like momenta, supercharges are not among the asymptotic observables of the supergravity theory. This is a consequence of the fact that there are no asymptotically covariantly constant spinors in such a conical geometry. In our case, we couple supersources (superparticles) to the Chern Simons supergravity in a super Poincaré invariant manner, so that our theory is not strictly a field theory. We find, not surprisingly, that the supersymmetry generators are again not among the asymptotic observables in our approach. We point out, however, that in analogy with the Poincaré Chern Simons gravity case [8], where the two asymptotic observables, i.e., mass and spin, are Casimir invariants of the Poincaré group, the asymptotic observables of the super Poincaré Chern Simons supergravity must also be identified with the Casimir invariants of the super Poincaré group associated with an equivalent one-superbody state described in [5]. We show that the emerging supersymmetric space-time is characterized by these invariants. After establishing these results, we compare the physical consequences of the picture which emerges with what is expected in the framework of an observation by Witten $[9,10]$

To provide the reader with some background and to make contact with previous works, we begin with a brief discussion of supersources which are to be coupled to the Chern Simons action for the super Poincaré group and their invariants. It will be recalled [8] that in the case of Poincaré gravity the sources(particles) can be viewed as irreducible representations of the Poincaré group in the same way as these representations are used in particle physics in $3+1$ dimensions. Similarly, we take a superparticle(supersource) to be an irreducible representation of the super Poincaré group. From this point of view, a superparticle is an irreducible supermultiplet consisting of several Poincaré states related to each other by the action of the supersymmetry generators. Clearly, this can be done for a simple or an extended supersymmetry with or without central charges. But in the interest of explicitness, we will consider in detail the $N=2$ super Poincaré group. The $N=2$ super Poincaré 
algebra in $2+1$ dimensions can be written as [6]

$$
\begin{array}{lll}
{\left[J^{a}, J^{b}\right]=-i \epsilon^{a b c} J_{c}} & ; & {\left[P^{a}, P^{b}\right]=0} \\
{\left[J^{a}, P^{b}\right]=-i \epsilon^{a b c} P_{c}} & ; & {\left[P^{a}, Q_{\alpha}\right]=0} \\
{\left[J^{a}, Q_{\alpha}\right]=-\left(\sigma^{a}\right)_{\alpha}^{\beta} Q_{\beta}} & ; & {\left[P^{a}, Q_{\alpha}^{\prime}\right]=0} \\
{\left[J^{a}, Q_{\alpha}^{\prime}\right]=-\left(\sigma^{a}\right)_{\alpha}^{\beta} Q_{\beta}^{\prime}} & ; & \left\{Q_{\alpha}, Q_{\beta}\right\}=0 \\
\left\{Q_{\alpha}, Q_{\beta}^{\prime}\right\}=-\sigma_{\alpha \beta}^{a} P_{a} & ; & \left\{Q_{\alpha}^{\prime}, Q_{\beta}^{\prime}\right\}=0 \\
a=0,1,2 & ; & \alpha=1,2
\end{array}
$$

The indices of the two component spinor charges $Q_{\alpha}$ and $Q_{\alpha}^{\prime}$ are raised and lowered by the antisymmetric metric $\epsilon^{\alpha \beta}$ with $\epsilon^{12}=-\epsilon_{12}=1$. the $S O(1,2)$ matrices $\sigma^{a}$ satisfy the Clifford algebra

$$
\left\{\sigma^{a}, \sigma^{b}\right\}=\frac{1}{2} \eta^{a b}
$$

where $\eta^{a b}$ is the Minkowski metric with signature $(+,-,-)$. We also have

$$
\sigma_{\alpha \beta}^{a}=\left(\sigma^{a}\right)_{\alpha}^{\gamma} \epsilon_{\gamma \beta}
$$

It is convenient to take the matrices $\sigma^{a}$ to be

$$
\sigma^{0}=\frac{1}{2}\left(\begin{array}{cc}
1 & 0 \\
0 & -1
\end{array}\right) \quad ; \quad \sigma^{1}=\frac{1}{2}\left(\begin{array}{cc}
0 & i \\
i & 0
\end{array}\right) \quad ; \quad \sigma^{2}=\frac{1}{2}\left(\begin{array}{cc}
0 & 1 \\
-1 & 0
\end{array}\right)
$$

The two Casimir operators of the super Poincaré group are given by

$$
\begin{aligned}
& C_{1}=P^{2}=\eta^{a b} P_{a} P_{b} \\
& C_{2}=\eta^{a b} P_{a} J_{b}+\epsilon^{\alpha \beta} Q_{\alpha}^{\prime} Q_{\beta}
\end{aligned}
$$

The first of these is the same as the Casimir operator of the Poincare subgroup, so that its eigenvalues can be identified with the square of the mass of the superparticle. Since the Pauli-Liubanski operator (or its square) does not commute with supersymmetry transformations, it must be supplemented with the second term on the right hand side of equation (6) to obtain a super Poincaré invariant. We will designate its eigenvalues as $m c_{2}$.

Irreducible representations of the $N=2$ super Poincaré group in $2+1$ dimensions can be constructed along the same lines as those in $3+1$ dimensions [11]. We note that without loss of generality we can work in a frame in which the supermultiplet is at rest. Then the non-vanishing anti-commutators of the superalgebra simplify to

$$
\left\{Q_{1}, Q_{2}^{\prime}\right\}=\left\{Q_{2}, Q_{1}^{\prime}\right\}=\frac{m}{2}
$$

Thus $Q_{\alpha}$ and $Q_{\alpha}^{\prime}, \alpha=1,2$, form a Clifford algebra. We define a Clifford vacuum state , $\mid \Omega>$ by the requirement

$$
Q_{\alpha} \mid \Omega>=0 ; \alpha=1,2
$$


It is easy to verify that such a state exists within every supermultiplet and that it is an eigenstate of $C_{1}$ and $C_{2}$ :

$$
\begin{aligned}
& C_{1}\left|\Omega>=m^{2}\right| \Omega> \\
& C_{2}\left|\Omega>=m c_{2}\right| \Omega>
\end{aligned}
$$

From the definition of the Clifford vacuum state in the rest frame of the superparticle, it follows that

$$
\begin{aligned}
C_{2} \mid \Omega> & =P \cdot J \mid \Omega> \\
& =m s^{0} \mid \Omega> \\
& =m s \mid \Omega>
\end{aligned}
$$

where we identify the eigenvalue, $s$, of the operator $s^{0}$ with the spin of the state $\mid \Omega>$. So, the Clifford vacuum state is a Poincaré state with mass $m$ and spin $s$ :

$$
|\Omega>=| m, s>
$$

Consider, next, the states

$$
\begin{aligned}
& \left|\Omega_{1}>=Q_{1}^{\prime}\right| \Omega>, \\
& \left|\Omega_{2}>=Q_{2}^{\prime}\right| \Omega>
\end{aligned}
$$

and

$$
\left|\Omega_{12}>=Q_{1}^{\prime} Q_{2}^{\prime}\right| \Omega>
$$

It is easy to verify that

$$
\begin{aligned}
s^{0} \mid \Omega_{1}> & =\left(s-\frac{1}{2}\right) \mid \Omega_{1}> \\
s^{0} \mid \Omega_{2}> & =\left(s+\frac{1}{2}\right) \mid \Omega_{1}> \\
s^{0} \mid \Omega_{12}> & =s \mid \Omega_{12}>
\end{aligned}
$$

These three Poincaré states together with the Clifford vacuum state form an Irreducible supermultiplet of $N=2$ super Poincaré group in $2+1$ dimensions, which we call a superparticle. Each supermultiplet is distinguished by its mass $m$ and the eigenvalue $c_{2}=s$, where $s$ is the spin of the Clifford vacuum state. We will refer to $c_{2}$ as the superspin of the multiplet.

One interesting feature which distinguishes the representations of the Poincaré group in $2+1$ dimensions from those in $3+1$ dimensions is that in $2+1$ dimensions the little group of time-like momenta is $S O(2)$, so that the spin of a Poincaré state is not limited to integer and half integer values and can be any real number. The spins of the states within a supermultiplet are fixed once the value of $c_{2}$ is specified. 
For example, for $c_{2}=\frac{1}{2}$, the resulting $N=2$ supermultiplet is a vector multiplet consisting of a spin zero, two spin $1 / 2$, and one spin one Poincaré states.

For later use, we give here an explicit realization of the $N=2$ super Poincaré algebra by extending the phase space variables $p^{a}$ and $q^{a}$ of the Poincaré group to their supersymmetric form:

$$
p^{a} \longrightarrow p^{A} \equiv\left(p^{a}, p^{\alpha}\right) \quad ; \quad q^{a} \longrightarrow q^{A} \equiv\left(q^{a}, q^{\alpha}\right)
$$

In terms of these variables, the generators of the super Poincaré algebra take the form

$$
\begin{aligned}
P_{a} & =i \partial_{a} ; Q_{\alpha}=-\partial_{\alpha} ; Q_{\alpha}^{\prime}=i\left(\sigma^{a}\right)_{\alpha \beta} q^{\beta} \partial_{a} \\
J_{a} & =\epsilon_{a b c} q^{b} p^{c}+\left(\sigma_{a}\right)_{\alpha}^{\beta} q^{\alpha} \partial_{\beta}+s_{a}
\end{aligned}
$$

We now turn to the action of the two-superbody problem [5]. For the super Poincaré algebra, the connection can be written as

$$
A_{\mu}=e_{\mu}^{a} P_{a}+\omega_{\mu}^{a} J_{a}+\chi_{\mu}^{\alpha} Q_{\alpha}+\xi_{\mu}^{\alpha} Q_{\alpha}^{\prime}
$$

Then, the Chern Simons action for the super Poincaré group can be written as

$$
\begin{aligned}
I_{c s}= & \frac{1}{2} \int_{M}\left\{\eta_{b c}\left[e^{b} \wedge\left(2 d \omega^{c}+\epsilon_{d a}^{c} \omega^{d} \wedge \omega^{a}\right)\right]\right. \\
& \left.-\epsilon_{\alpha \beta}\left[\chi^{\alpha} \wedge\left(d-i \sigma_{a} \omega^{a}\right) \psi^{\beta}+\psi^{\alpha} \wedge\left(d-i \sigma_{a} \omega^{a}\right) \chi^{\beta}\right]\right\}
\end{aligned}
$$

This action is invariant under the local infinitesimal gauge transformations

$$
\delta A_{\mu}=\partial_{\mu} u+i\left[A_{\mu}, u\right]
$$

where

$$
u=\rho^{a} P_{a}+\tau^{a} J_{a}+\nu^{\alpha} Q_{\alpha}+\nu^{\prime \alpha} Q_{\alpha}^{\prime}
$$

As in the case of Poincaré gravity [8], the manifold $M$ is specified by its topology and is not to be identified with space-time which will emerge (see below) as an output of this gauge theory.

To couple (super)sources to this Chern Simons theory, we proceed in a manner similar to the way sources were coupled to the Poincaré Chern Simons theory. From the discussion of the supermultiplets given above, we conclude that the logical candidates for our supersources are the irreducible representations of the $N=2$ super Poincaré group. Then each supersource can be coupled to the $N=2$ Chern Simons supergravity by an action of the form [5]

$$
\begin{array}{r}
I_{s}=\int_{C} d \tau\left\{p_{a} \partial_{\tau} q^{a}-\epsilon_{\alpha \beta} p^{\alpha} \partial_{\tau} q^{\beta}-t^{\mu}\left(e_{\mu}^{a} p_{a}+\omega_{\mu}^{a} j_{a}-i \epsilon_{\alpha \beta} \chi_{\mu}^{\alpha} p^{\beta}\right.\right. \\
\left.\left.+(\sigma \cdot p)_{\alpha \beta} \xi_{\mu}^{\alpha} q^{\beta}\right)+\lambda_{1}\left(p^{2}-m^{2}\right)+\lambda_{2}\left(c_{2}-s\right)\right\}
\end{array}
$$

where $\tau$ is an invariant parameter along the trajectory $C$. Also, $m c_{2}$ is an eigenvalue of the second Casimir operator of the super Poincaré group, and $s$ is the spin of the 
Clifford vacuum state of the supermultiplet. The choice of the constraint multiplying $\lambda_{2}$ is crucial in relating the eigenvalue of the second Casimir invariant, $c_{2}$, of the superalgebra to the spin content of a supermutiplet. For more than one source, one can add an action of this type for each source. In the presence of supersources the topology of the manifold is modified. But the field strengths still vanish outside supersources, and the theory is locally trivial.

It was shown in reference [5] that the exact gauge invariant observables of the twosuperbody system can be obtained in terms of Wilson loops. They may be viewed as the Casimir invariants of an equivalent one-superbody state, as was done in Chern Simons gravity [8]. We will refer to these invariants as $H$ and $C_{2}$. As we have seen above, their eigenvalues determine mass(energy) and spin(angular momentum) content the supermultiplet. They constitute the asymptotic observables of the twosuperbody system. Here we simply quote the expressions from which $H$ and $C_{2}$ can be computed exactly $[5,6]$. Since the invariant $H$ is the same as that for the Poincaré algebra, it is given by [8]

$$
\cos \frac{H}{2}=\cos \frac{m_{1}}{2} \cos \frac{m_{2}}{2}-\frac{p_{1} \cdot p_{2}}{m_{1} m_{2}} \sin \frac{m_{1}}{2} \sin \frac{m_{2}}{2}
$$

To obtain $C_{2}$, define the quantity $\left|Z_{ \pm}\right|$according to

$$
\left|Z_{ \pm}\right|=\left[H^{2} \pm 2 C_{2}\right]^{\frac{1}{2}}
$$

Also define

$$
W_{R}\left(C_{0}\right)=\left(2 \cos \frac{\left|Z_{+}\right|}{2}-1\right)\left(2 \cos \frac{\left|Z_{-}\right|}{2}-1\right)
$$

Then in terms of the invariants of the two-superbody system we have

$$
W_{R}\left(C_{0}\right)=W_{x} W_{y}
$$

where

$$
\begin{aligned}
W_{x}= & \left\{1+2\left(\cos \frac{\left|x_{1}\right|}{2}-1\right)+2\left(\cos \frac{\left|x_{2}\right|}{2}-1\right)\right. \\
& -2\left(\left|x_{1}\right|\left|x_{2}\right|\right)^{-1}\left(x_{1}^{a} \cdot x_{2}^{a}-\epsilon_{\alpha \beta} q_{1}^{\alpha} q_{2}^{\beta}\right) \sin \frac{\left|x_{1}\right|}{2} \sin \frac{\left|x_{2}\right|}{2} \\
& +\left(\left|x_{1}\right|\left|x_{2}\right|\right)^{-2}\left(\cos \frac{\left|x_{1}\right|}{2}-1\right)\left(\cos \frac{\left|x_{2}\right|}{2}-1\right) \\
& \times\left[\left|x_{1}\right|^{2}\left|x_{2}\right|^{2}+4 i \epsilon_{a b c} x_{1}^{a} x_{2}^{b}(\sigma)_{\alpha \beta} q_{1}^{\alpha} q_{2}^{\beta}+\left(x_{1}^{a}\right)^{2}\left(x_{2}^{a}\right)^{2}\right. \\
& \left.\left.\quad-2 x_{1}^{a} x_{2}^{a} \epsilon_{\alpha \beta} q_{1}^{\alpha} q_{2}^{\beta}+\epsilon_{\alpha \beta} \epsilon_{\gamma \delta} q_{1}^{\alpha} q_{2}^{\gamma} q_{1}^{\beta} q_{2}^{\delta}\right]\right\}
\end{aligned}
$$

In this expression, with $k=1,2$, and $x^{A}=\left(x^{a}, q^{\alpha}\right)$, we have

$$
x_{k}^{a}=p_{k}^{a}+j_{k}^{a} \quad ; \quad q_{k}^{\alpha}=p_{k}^{\alpha}+j_{k}^{\alpha}
$$


The factor $W_{y}$ has exactly the same structure as $W_{x}$ with $x^{A} \longrightarrow y^{A}=\left(y^{a}, q^{\prime \alpha}\right)$, where

$$
y_{k}^{a}=p_{k}^{a}-j_{k}^{a} \quad ; \quad q_{k}^{\alpha}=p_{k}^{\alpha}-j_{k}^{\alpha}
$$

Having obtained the gauge invariant observables of the two- superbody system, we now turn to the structure of the corresponding space-time. In studying the properties of this space-time, we are guided by the space-time structure which emerged from the dynamics of the two-body system in Poincare Chern Simons gravity [8]. There it was shown that, by a suitable choice of gauge, the spacial components of the relative phase space variable $q$ of the two-body system specify a cone with the deficit angle $H$ given by equation (26). In terms of the gauge fixed variables, aside from the specific significance of $H$ and $s$ in the present context, the expression for the line element has the same form as is known for any spinning cone [12] :

$$
d s^{2}=d q_{0}^{\prime 2}-d r^{2}-r^{2} d \phi^{\prime 2}
$$

Or in terms of more familiar coordinates

$$
d s^{2}=\left(d q^{0}-\frac{s d \phi}{2 \pi}\right)^{2}-d r^{2}-\alpha^{2} r^{2} d \phi^{2}
$$

The coordinates in these equivalent expressions are related by

$$
q^{\prime 0}=q^{\prime 0}\left(q^{0}, \phi^{\prime}\right)=q^{0}-\frac{s \phi^{\prime}}{2 \pi \alpha}
$$

and

$$
\overrightarrow{q^{\prime}}=\left[\exp i \tau^{0} J_{0}\right] \vec{q}
$$

where

$$
\tau^{0}=\left(1-\frac{H}{2 \pi}\right) \equiv \alpha \phi=\phi^{\prime}
$$

In the supersymmetric case, the situation is somewhat more complicated. To see why, we note that in both cases we can associate our gauge invariant observables to a reduced one-(super)body state. In the pure gravity case, such a state is a single Poincaré state, but in the supersymmetric case it is a supermultiplet consisting of several (four for $N=2$ ) Poincaré states. As stated above, in the case of Poincaré Chern Simons theory, the structure of the emerging space-time and its asymptotic observables are completely determined by the numerical values of the two (gauge invariant) Casimir invariants of the reduced one-body Poincaré state. To see how this picture generalizes for the two-superbody system, we recall that our two supersources are characterized by charges $\left(p_{1}^{A}, j_{1}^{A}\right)$ and $\left(p_{2}^{A}, j_{2}^{A}\right)$ with the corresponding canonical coordinates $q_{1}^{A}$ and $q_{2}^{A}$, respectively. Without loss of generality, let the first supersource be at rest at the origin, i. e. , $\vec{q}_{1}=0$. Then $\vec{q}_{2} \equiv \vec{q}$ can be viewed as a relative coordinate. As in pure gravity, we parametrize $\vec{q}$ by its polar components: $\vec{q}=(r, \phi)$. By fixing $\vec{q}_{1}=0$, we have again made a choice of gauge which fixes all the $N=2$ 
super Poincaré gauge transformations except for the spatial rotations generated by $J^{0}$ and translations along $q^{0}$. To fix these, consider first the same transformation as specified by equations (36) and (37). Being an element of $N=2$ super Poincaré group, this transformation leaves the Casimir invariants $H$ and $c_{2}$ unchanged. But again the $\overrightarrow{q^{\prime}}$ is no longer $2 \pi$ periodic and satisfies the matching conditions for the coordinates on a cone characterized by the deficit angle $\beta=H$.

Up to this point, everything looks the same as in Poincaré gravity space-time. However, essential differences appear when we try to gauge fix the translations along $q^{0}$. It will be recalled from our discussion of supersources that an $N=2$ supermultiplet at rest with Casimir invariants $H$ and $c_{2}$ consists of four Poincaré states with the following spin eigenvalues :

$$
\begin{aligned}
& P \cdot J\left|H, c_{2}, s_{1}>=H\left(c_{2}-\frac{1}{2}\right)\right| m, c_{2}, s_{1}> \\
& P \cdot J\left|H, c_{2}, s_{2}>=H c_{2}\right| m, c_{2}, s_{2}> \\
& P \cdot J\left|H, c_{2}, s_{3}>=H c_{2}\right| m, c_{2}, s_{3}> \\
& P \cdot J\left|H, c_{2}, s_{4}>=H\left(c_{2}+\frac{1}{2}\right)\right| m, c_{2}, s_{4}>
\end{aligned}
$$

Let us compare this supermultiplet with the Poincaré state obtained in the reduction of Poincaré Chern Simons gravity. In the latter case, it was possible to further fix the gauge in $q^{0}$ direction by the transformation (35) which involved the spin of the Poincaré state. Clearly, this is no longer possible for a supermultiplet consisting of Poincaré states of different spins. This makes it impossible for a single metric of the form (34) or (35) to describe all the spin states of our equivalent one-superbody multiplet. So, to describe all the spin states corresponding to our gauge invariant observables $H$ and $c_{2}$, we generalize the usual notion of a line element to an "operator line element" which acts on the supermultiplet of Poincaré states. This operator has the form

$$
d s^{2}=\left(d q^{0}-\frac{S d \phi}{2 \pi}\right)^{2}-d r^{2}-\alpha^{2} r^{2} d \phi^{2}
$$

where now $S=\frac{P \cdot J}{H}$ is the spin operator of the supersymmetry algebra. We could also regard $H$ and hence $\alpha$ as an operator in the expression (42). But since $H$ is a Casimir operator and has the same eigenvalue for all the states of a supermultiplet, we may replace it with its eigenvalue throughout. When this operator line element acts on a state of a supermultiplet, we can replace $S$ with the spin eigenvalue of that state and hence specify the corresponding space-time. It therefore follows that the description of all the spin states of the equivalent one-body supermultiplet requires a multiplet of space-times equal in number to the dimension of the supermultiplet (four for $N=2$ ). The line elements for the members of this space-time multiplet, with $k=1, . ., 4$, are given by

$$
d s_{k}^{2}=\left(d q^{0}-\frac{s_{k} d \phi}{2 \pi}\right)^{2}-d r^{2}-\alpha^{2} r^{2} d \phi^{2}
$$


The line element operator in equation (42) is not invariant under supersymmetry transformations, and it transforms in the same way as the Poincaré states within a supermultiplet. In other words, for $k=1, . ., 4$ the line elements in equation (43) form an irreducible representation of the $N=2$ supersymmetry and are completely determined by the asymptotic observables $H$ and $C_{2}$. Thus, the space-time description of the two-super- body system coupled to the super Poincaré Chern Simons action requires not just one but a supermultiplet of space-times. One way to view this supersymmetric space-time is to regard it as an ordinary space-time with an additional finite discrete dimension. It is also interesting to note that we can still write the metric operator (42) in same form as (33) by defining

$$
q^{\prime 0}=q^{0}-\frac{S \phi}{2 \pi}
$$

But then it is clear that the coordinates of such a generalized conical geometry will no longer be c-numbers and will not commute with each other.

It will be instructive to compare our results with the conclusions of Henneaux who analyzed metrical supergravity in $2+1$ dimensions [7]. Assuming that the spacetime is asymptotically conical, he showed that there are no Killing spinors associated with the supersymmetric generators so that the supercharges cannot be asymptotic observables. From our point of view, the asymptotic observables of the Chern Simons supergravity coupled to (super)matter are the Casimir invariants $\left(H, c_{2}\right)$ which label the equivalent one-superbody state. It is, therefore, not surprising that there are no observables associated with (odd) supersymmetry generators. It is the invariant $c_{2}$ which is an asymptotic observable and signals the presence of supersymmetry. Moreover, as we have seen, to realize the local supersymmetry, one needs not just one conical space-time but a supermultiplet of them spanning an irreducible representation of $(N=2)$ supersymmetry. The supersymmetry generators act as ladder operators relating the spinning cones within the space-time supermultiplet.

Let us consider some physical consequences of the supersymmetric space-time which emerges from the above analysis and compare them with Witten's observations [9]. Although both pictures arise in $2+1$ dimensions, it is conceivable that one or both might have $3+1$ dimensional analogues. Drawing on the specific features of the conical geometry mentioned above, Witten pointed out the interesting possibility that in $2+1$ dimensions one can, in principle, arrange for the vanishing of the cosmological constant without having the equality of masses for members of a supermultiplet. In the supersymmetric space-time which emerges from our formalism, supersymmetry remains exact, and particles in a supermultiplet will have the same mass. However, the superpartners will reside in different members of the space-time supermultiplet, thus making their direct detection non-trivial. From this point of view, any hope of detecting the superpartners of the known particles will rest on supersymmetry remaining unbroken. Otherwise, the superpartners will end up in disconnected universes. We emphasize that such supersymmetric worlds may turn 
out to be peculiar to $2+1$ dimensions only. But still it would be of interest to see how one can experimentally establish or rule out the existence of such a superworld. We hope to address this question elsewhere.

We would like to thank F. Ardalan for discussions and helpful suggestions. This work was supported in part by the Department of Energy under the contract No. DOE-FG02-84ER40153.

\section{References}

1. A. Achucarro and P.K. Townsend, Phys. Lett B180 (1986) 89

2. E. Witten, Nucl. Phys. B311 (1988) 46 and B323 (1989) 113

3. K. Koehler, F. Mansouri, C. Vaz, L. Witten, Mod. Phys. Lett. A5(1990) 935

4. K. Koehler, F. Mansouri, C. Vaz, L. Witten, Nucl. Phys. B341 (1990) 167 andB348 (1990) 373

5. Sunme Kim and F. Mansouri, Phys. Lett. B 372(1990) 72

6. A preliminary version of our results were reported at The International Conference on Seventy Years of Quantum Mechanics and Modern Trends in Theoretical Physics, Calcutta, India 1/29- 2/2/1996, to appear in the Proceedings, ed. P. Bandyopadhyay

7. M. Henneaux, Phys. Rev. D29 (1984) 2766

8. F. Mansouri and M.K. Falbo-Kenkel, Mod. Phys. Lett. A8 (1993) 2503; F. Mansouri, Comm. Theo. Phys. 4 (1995) 191

9. E. Witten, Int. Jour. Mod. Phys. A10 (1995) 1247

10. K. Becker, M. Becker, A. Strominger, Phys. Rev. D51 (1995) R6603

11. A. Salam and J. Strathdee, Forts. Phys. 26 (1978) 57; P.G.O. Freund, Introduction to Supersymmetry, Cambridge University Press, 1986

12. S.Deser, R.Jackiw, G. 't Hooft, Ann. of Phys. (N.Y.) 152 (1984) 220; S. Giddings, J. Abbott and K. Kuchar, Gen. Rel. Grav. 16(1984) 751; J.R. Gott and M. Alpert, Gen. Rel. Grav. 16 (1984) 751 